\title{
Multidisciplinary treatment planning in elderly patients with cancer: a prospective observational study
}

\author{
Remei Blanco, PhD, ${ }^{a}$ Mercè Capo, MD, Anna Maria Libran, MD, ${ }^{c}$ Jordi Alfaro, MD, ${ }^{a}$ \\ Emma Dotor, MD, ${ }^{a}$ Luis Fernandez, PhD, ${ }^{a}$ Danae Guerra, MD, ${ }^{a}$ Esther Jovell, PhD, ${ }^{d}$ \\ Mireia Llonch, MD, ${ }^{\mathrm{b}}$ Aleydis Pisa, MD, and Angels Arcusa, $\mathrm{MD}^{\mathrm{a}}$
}

Departments of ${ }^{a}$ Medical Oncology, ${ }^{b}$ Geriatrics, and ${ }^{c}$ Palliative Care, and the ${ }^{\mathrm{d}}$ Technical Research Office, Consorci Sanitari de Terrassa, Terrassa, Spain

\begin{abstract}
Background Elderly cancer patients are a special population, and their management should include specialists in oncology, geriatrics, palliative care, and social work. Based on this approach, we designed a multidisciplinary care model (MCM) and prospectively assessed its results.
\end{abstract}

Objectives To evaluate the applicability of the MCM, to describe the geriatric features of our sample, and to assess the impact of the MCM on treatment choices.

Methods Patients older than 69 years of age with solid tumours were included. The MCM included the following decision algorithm: Patients with an unequivocal condition of frailty, assessed in the corresponding tumor committee, were directly referred to the palliative care team (Group A). In the other

cases (Group B), patients over age 79 years underwent the Comprehensive Geriatric Assessment (CGA) and patients aged between 70 and 79 years completed a frailty test. If the frailty test was positive, CGA was also performed.

Results 295 patients meeting the inclusion criteria were identified during one year. $186(63 \%)$ were included in the MCM.. A total of 66 CGA were performed. CGA modified the therapeutic plan in 5 patients older than $80(13.8 \%)$, and in 2 septuagenarian patients $(6.6 \%)$.

Limitations This study was designed to evaluate the feasibility of a multidisciplinary approach in geriatric oncology patients in a real clinical setting. Therefore, some variables were not fully controlled in the design, such as the willingness of different specialists to refer their patients to the model.

Conclusions MCM in elderly oncology patients is feasible in a general hospital, although several reasons often hinder patient recruitment for this kind of program. CGA can modify the therapeutic plan, especially in the octogenarian population.

Funding/sponsorship This study has been financially supported by a grant from the Fundació Joan Costa Romà.

A $\mathrm{s}$ in many European countries and in North America, the Spanish population continues to age. ${ }^{1}$ In January $2014,18.1 \%$ of the Spanish population was composed of people older than 65 , and octogenarians now represent $5.7 \%$ of the entire population. The predominant sex among the elderly is female: there are $34 \%$ more women than men. In addition, cancer is often a disease of the elderly. ${ }^{2,3}$ More than $65 \%$ of all malignant tumors affect this population. In Spain, cancer is the second leading cause of death, after cardiovascular diseases (83,115 cancer deaths in 2012). Physiological ageing entails a particular set of characteristics that the oncologist should take into consideration, such as neuropsychological changes (depression, disso- ciative symptoms, sensory alterations, and memory loss), ${ }^{4}$ unfavorable social and family environments, polymedication (with increased risks of pharmacological interactions), ${ }^{5}$ pharmacokinetic changes, ${ }^{6}$ and a decrease in medullary cellularity with higher risk of toxicity from chemotherapy.

The therapeutic approach to an elderly patient with cancer will vary according to tumor type, functional status, and comorbidity. Frailty often precludes the use of specific oncological treatment. ${ }^{7,8}$ In such cases, symptomatic treatment and comfort care are usually appropriate. Palliative care is therefore often offered to frail elderly patients. It should nevertheless be borne in mind that age per se is not a contraindication for specific oncological treatments. ${ }^{9}$

Accepted for publication September 30, 2016. Correspondence: Remei Blanco, PhD; rblanco@cst.cat. Disclosures: The authors report no disclosures or conflicts of interest. JCSO 2016;14(11):466-472. @2016 Frontline Medical Communications. doi: 10.12788/jcso.0299. 
Comprehensive geriatric assessment (CGA) is a multidimensional interdisciplinary diagnostic process designed to identify the physical, functional, cognitive, and social problems faced by the elderly in order to tailor treatment and follow-up plans. ${ }^{10-13}$ The impact of CGA on treatment decision-making in oncological clinical practice has been questioned. ${ }^{14}$ CGA is time-intensive and cumbersome but, for the moment, it remains the gold standard in the treatment decision process for elderly patients. However, a number of screening tools have been suggested for the initial assessment of elderly patients, ${ }^{15,16}$ in an attempt to rationalize health resources and reserve CGA for patients who really need a more detailed assessment.

Based on that rationale, we implemented a Multidisciplinary Care Model (MCM) and an Oncogeriatric Multidisciplinary Committee in our institution, composed of oncologists, radiotherapists, geriatricians, palliative specialists, psycho-oncologists and specialized nurses. Data on the procedures were gathered a year later. Our aims were to assess the feasibility of the MCM; describe the geriatric variables of patients undergoing CGA - comorbidities, polypharmacy, and functional, cognitive, nutritional, and social deficits; and evaluate the impact of the MCM on final treatment decisions.

\section{Methods}

The study was designed as a prospective, observational study for collecting information during the course of a year from patients who were older than 69 years and had been diagnosed with malignant solid tumors at the Consorci Sanitari de Terrassa hospital in Terrassa, Spain. Patients with superficial urinary bladder tumors were excluded.

The MCM consisted of the following algorithm: Each case was initially discussed in the appropriate Tumor Committee (thoracic tumors, urological tumors, breast cancer, gastrointestinal cancers, and gynecological cancers). These committees were composed of oncologists, pathologists, radiologists, radiotherapists, and a different surgeon in each case, that is, a thoracic surgeon, a gynecologist, a urologist, and so on. Other specialists joined each committee depending on the topic (gastroenterologists, pulmonologists). (Figure 1)

At this baseline evaluation, some patients were immediately referred to the Palliative Care department for onco-specific treatment, because of major comorbidities or a poor performance status (Group A). The remaining patients (Group B) received an initial treatment plan proposed by the Tumor Committees and, thereafter, were divided into 2 groups: those older than 79 years underwent a CGA before a definitive treatment decision was made; and those aged 70-79 years were screened with a frailty test (FT). ${ }^{17}$ Our FT had been validated for the Spanish population, showing a sensitivity of $83 \%$ and a specificity of $50 \%$, with respect to CGA. It was administered by a trained nurse, and consisted of a 1-sheet questionnaire

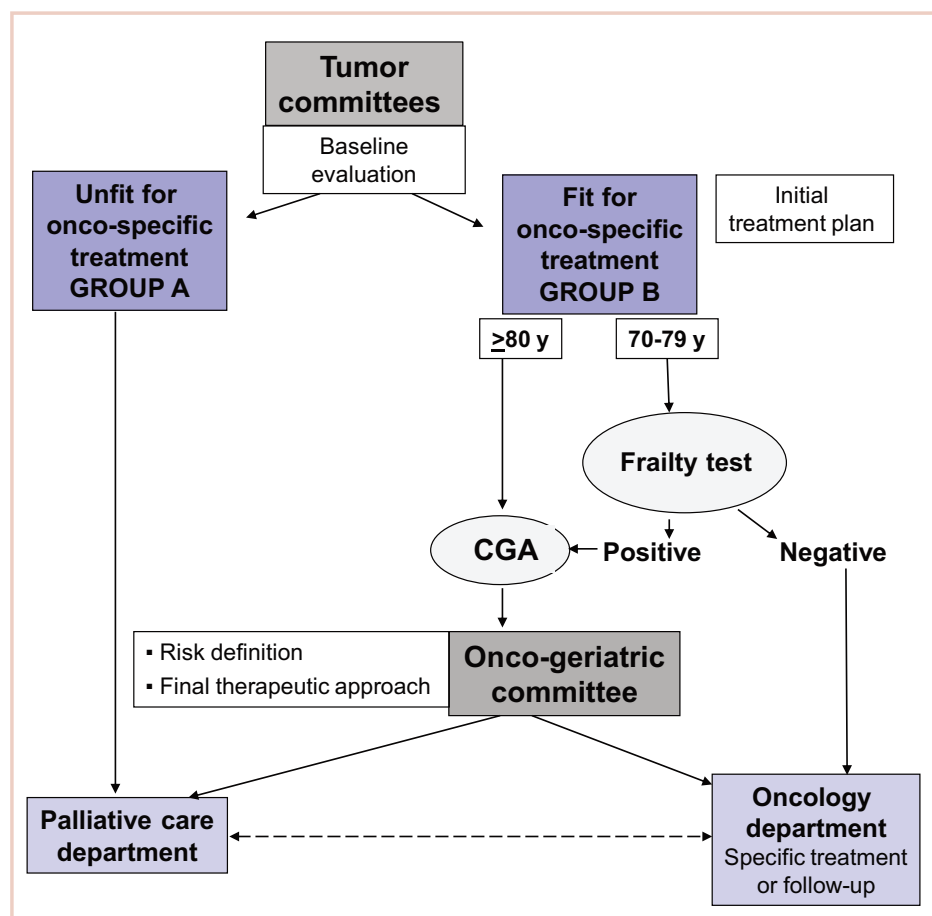

FIGURE Oncology patients older than 69 years of age. Patient flow in the multidisciplinary care model.

containing 10 questions that explored functional, cognitive, social, and nutritional status (Table 1 [English translation of the frailty test]). The final result was positive for frailty if there were $\geq 2$ frailty criteria present. Patients with a positive FT were also referred for CGA before a final treatment decision was made. Patients with a negative FT underwent the initial treatment plan. The CGA was performed by a geriatrician, and the following features were evaluated:

Comorbidity, using the Charlson Comorbidity Index (CCI). ${ }^{18}$ This scoring system evaluates 19 items with different ratings. For example, Peripheral Vascular Disease is allocated 1 point, whereas Hemiplegia is assigned 2 points. Depending on the patient's age, $0-3$ points are added to the total count. Using established formulae, the Charlson index and the probability of survival at 10 years are calculated.

Polypharmacy, based on the STOPP (Screening Tool of Older Person's Prescriptions) and START (Screening Tool to Alert doctors to Right Treatment) criteria. ${ }^{19}$

Functional status, using the following scales:

- The Lawton Instrumental Activities of Daily Living (IADL), ${ }^{20}$ which assesses a person's ability to perform tasks such as using a telephone, doing laundry, and handling finances. The scale may provide an early warning of functional decline or signal the need for further assessment. 


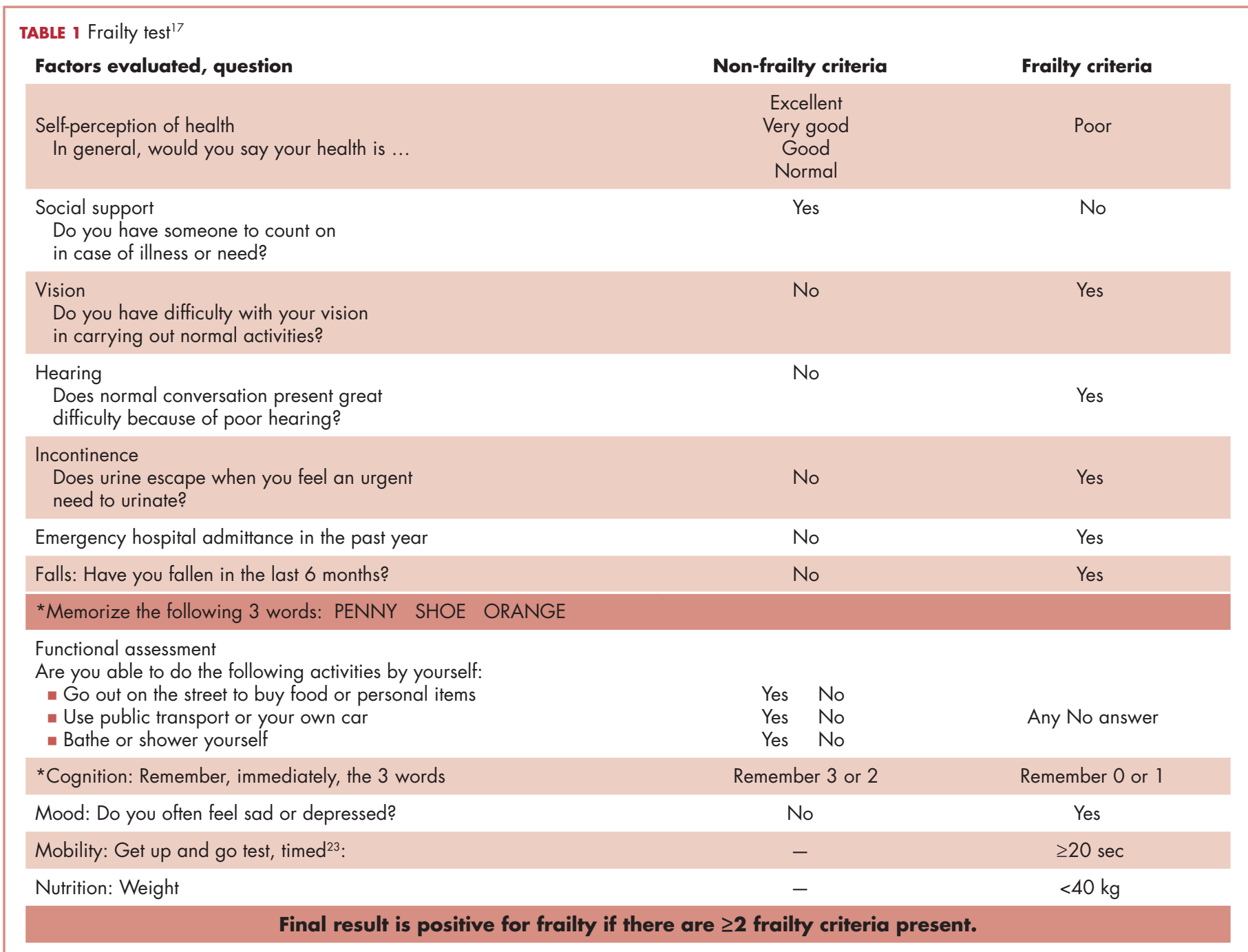

- The Barthel Index, ${ }^{21}$ consisting of 10 items that measure a person's daily functioning, particularly the activities of daily living (ADL) and mobility. The items include feeding, transfers from bed to wheelchair, using the toilet, grooming, walking on a level surface, going up and down stairs, dressing, and continence of bowels and bladder.

- The Timed Up and Go test (TUG), ${ }^{22}$ for assessing the time a person takes to rise from a chair, walk 3 meters, turn around, walk back to the chair, and sit down.

- The Short Physical Performance Battery (SPPB), ${ }^{23}$ a group of measures that combines the results of the gait speed, chair stand, and balance tests.

Cognitive and emotional condition, based on the following tools:

- The Mini-Mental State Examination (MMSE), ${ }^{24}$ a 30-point questionnaire, taking between 5-10 minutes, that examines functions including registration, atten- tion and calculation, recall, language, ability to follow simple commands and orientation.

- The Clock Drawing test ${ }^{25}$ is used for screening for cognitive impairment and dementia, and as a measure of spatial dysfunction and neglect.

- The Geriatric Depression Scale $(\text { GDS-15) })^{26}$ is a 30-item self-report assessment used to identify depression in the elderly.

Nutritional status, using the Mini Nutritional Assessment $(\mathrm{MNA})^{27}$ tool that allows for identification of elderly patients suffering from, or at risk of, malnutrition. It consists of 6 differential questions (screening), and 12 additional questions that need only be asked of those individuals identified by the screening as possible cases of malnutrition.

Once the CGA had been performed, the Oncogeriatric Multidisciplinary Committee met every week to discuss 
the results. All of the patients submitted to CGA were discussed again and a final treatment approach was decided.

All of the patients were offered an assessment and monitoring by the palliative care team, whether a specific cancer treatment was planned or not. The palliative care team intervention consisted of a first assessment offered to all patients within the first month after diagnosis. Further monitoring was tailored according to the needs of each patient. On the first visit, the patient was assessed by a physician and nurse who were specialists in palliative care. The patient's symptoms were identified and appropriate treatments were recommended. A more or less frequent office follow-up was designed, depending on the symptom burden of each patient. In some cases, home care was preferred. Direct telephone contact with the palliative care team was also offered. Finally, the need for social worker intervention was considered, depending on the functional status and family context of each patient.

The following data were collected, in addition to the information provided by the CGA: demography, tumor site, inclusion or not in the MCM, screening or not with the FT and FT result, initial treatment plan mapped out by the Tumor Committees, and final treatment administered including symptomatic treatment. No statistical analysis was performed in this study, because our goal was only to describe the care model. All of the participants authorized the procedures in the MCM. However, written consent was not collected, because there were no interventions other than standard health care procedures. The study protocol was approved by the ethical committee on human research of the Consorci Sanitari de Terrassa.

\section{Results}

We identified 295 patients who met the inclusion criteria, of whom 170 were men and 125 were women. Patient characteristics with regard to age group and diagnosis are described in Table 2. The most frequent diagnosis among our patients was lung cancer (35 patients), followed by colon cancer (32 patients). A comparison of the 2 age groups (7079 years and 80 years or older) highlighted the higher prevalence of breast cancer and prostate cancer ( 21 vs 4 , and 14 vs 2 patients, respectively) among younger patients. For the rest of the diagnoses, there did not seemr to be significant differences in relation to age group, although the sample size does not allow for definitive conclusions.

\section{Feasibility of the MCM}

There were 295 patients meeting the inclusion criteria, of whom 186 were included in the MCM. So the feasibility of our care model could be quantified at $63 \%$. The major reasons for not enrolling were: non-referral by the responsible physician (83 patients, 28.1\%), need for prompt treatment start and/or delay in CGA (12 patients, 4\%), patient refusal to undergo CGA (6 cases, $2 \%$ ), treatment in a different hospital (5 patients, $1.7 \%$ ), early disease progression or death (2 patients, $0.6 \%$ ), and lack of continuity within the MCM (1 patient, $0.3 \%)$.

Patient flow in the MCM was as follows: 52 of 186 patients $(27.9 \%)$ were referred directly to the palliative care team (Group A), and the remaining 134 were initially considered for onco-specific treatment (Group B). Of the 134 patients, 36 who were older than 79 years underwent a CGA, and 98 patients aged between 70 and 79 years completed the frailty test; 30 patients had a positive test and were also referred for CGA. Thus, a total of 66 CGAs were performed. All but one of the 66 patients were discussed again in the weekly Oncogeriatric Multidisciplinary Committee and a final treatment pronouncement was taken. For the remaining patient, his responsible physician made a therapeutic decision without having discussed the case in the committee.

\section{Geriatric description of the population}

According to the scales we used, our 66 patients undergo-

TABLE 2 Primary tumor by age group

Age group, y (n)

\begin{tabular}{|c|c|c|c|}
\hline \multirow[b]{2}{*}{ Primary tumor } & \multirow[b]{2}{*}{$70-79(169)$} & \multirow[b]{2}{*}{$\geq 80$ (126) } & \multirow{2}{*}{$\begin{array}{l}\text { Total no. patients } \\
\quad(\mathbf{N}=\mathbf{2 9 5})\end{array}$} \\
\hline & & & \\
\hline Lung & 19 & 16 & 35 \\
\hline Breast & 21 & 4 & 25 \\
\hline Colorectal & 14 & 18 & 32 \\
\hline Other digestive & 20 & 17 & 37 \\
\hline Prostate & 14 & 2 & 16 \\
\hline Urothelial & 4 & 2 & 6 \\
\hline Gynecologic & 7 & 2 & 9 \\
\hline Other & 18 & 10 & 28 \\
\hline
\end{tabular}


ing CGA had no dependency at all in $62.7 \%$ of the cases, whereas their dependency was low-moderate in $23.25 \%$, moderate-severe in $12.7 \%$, and high in $1.16 \%$ of the cases. Most of our patients had no (67.4\%) or mild (17.4\%) cognitive impairment. With regard to nutritional status, in $63.9 \%$ of our patients it was optimal, and only $3.48 \%$ of patients had low, moderate, or severe malnutrition. Nutritional condition was not evaluated in $32.5 \%$ of the cases. Of our patients undergoing CGA, 59\% were receiving polypharmacy, according to STOPP and START criteria, and $41 \%$ were not. Comorbidity was defined as low in $21 \%$, and severe in $79 \%$ of our patients. Detailed comorbidities are described in Table 3. Finally, suitable social support was noted in $94 \%$ of the subjects.

\section{Impact of MCM on the final therapeutic decision:}

MCM modified the initial therapeutic plan in 5 patients who were older than $80(13.8 \%)$ and in 2 frail septuagenarian patients (6.6\%). In almost all cases, the CGA allowed for detection of geriatric syndromes that identified patients as more fragile than the initial evaluation results had indicated. For that reason, aggressive therapeutic proposals, which carry an unacceptable risk of morbidity and mortality, were discounted. In one case, the CGA also detected insufficient family support that further compromised the viability of the initial therapeutic proposals. Table 4 shows details of initial treatment plan and final treatment administered after inclusion of these patients in the MCM.

\section{Discussion}

Our first aim was to assess the applicability of the MCM in our elderly patients with cancer, given the logistical difficulties of implementing a multidisciplinary model for health professionals in a hospital environment. One third of the candidates were not included in the model. It should be emphasized that in 26 cases, there was a justified cause to exclude patients from the planned intervention: require-

\begin{tabular}{|c|c|}
\hline Comorbidity & $\%$ \\
\hline Cerebrovascular disease & 11 \\
\hline Diabetes & 25 \\
\hline Chronic pulmonary disease & 24 \\
\hline Cardiopathy & 28 \\
\hline Dementia & 9 \\
\hline Peripheral arteriopathy & 6 \\
\hline Kidney dysfunction & 6 \\
\hline Hepatopathy & 2 \\
\hline
\end{tabular}

ment for rapid treatment start, patient's refusal, treatment in a different hospital, and early disease progression or death. However, a worrying feature was the low awareness and low sensitization of some professionals to the project, which accounted for 83 patients (28\%) not being referred to the model. It should nevertheless be kept in mind that 36 of those 83 patients had squamous cell carcinoma of the skin. In most of the cases, the treatment consisted of surgical resection that did not require admission or general anesthesia. Consequently, geriatric assessment and the intervention of a multidisciplinary team were not indicated in these patients. Presently, such patients are no longer offered a geriatrician assessment in our center, except in specific cases. Thus, only 47 patients ( $15.9 \%$ of the 295 considered fit for inclusion) did not enter the MCM without a justified cause. To address this deficiency, there needs to be an increased awareness among health professionals about the benefits of CGA in elderly oncologic patients. The recent creation of an oncogeriatric work group within the Spanish Society of Medical Oncology could perhaps be useful in this matter. As to the greater prevalence of patients with breast cancer or prostate cancer in the group of younger

TABLE 4 Impact of comprehensive geriatric assessment on initial therapeutic plan

\begin{tabular}{|c|c|c|c|}
\hline \multirow{2}{*}{ Age group, y } & \multirow{2}{*}{ Cancer diagnosis } & \multicolumn{2}{|c|}{ Therapeutic plan } \\
\hline & & Initial & Final \\
\hline \multirow[t]{4}{*}{$70-79$} & Pancreatic (T3NO) & Palliative CT & Symptomatic tx \\
\hline & NSCLC (T3N2) & Curative RT & Symptomatic tx \\
\hline & NSCLC* (T2N2MO) & Curative surgery & Palliative CT \\
\hline & Colon cancer (in situ) & Curative surgery & Symptomatic TC \\
\hline \multirow[t]{3}{*}{$\geq 80$} & Rectal (pT3pNOMO) & $C T+R T$ and curative surgery & Curative surgery \\
\hline & Colon (pT3 pN2MO) & Adjuvant CT & Follow-up \\
\hline & Bladder (pT2NOMO & Curative RT & Symptomatic tx \\
\hline
\end{tabular}

CT, chemotherapy; NSCLC, non-small-cell lung cancer; RT, radiotherapy; TC, control and follow-up; tx, treatment 
patients, we believe that this finding can be explained by the type of treatments offered to elderly patients with these diagnoses. They are usually well-tolerated hormonal treatments, and as such the attending physician could be less motivated to seek a geriatric evaluation for these patients.

Regarding the geriatric description of the 66 patients undergoing the CGA, we detected that our population sample had a good previous functional, nutritional and cognitive status. However, it must be kept in mind that this was a preselected group of elderly patients. First, $27.9 \%$ of the participants included in the MCM had been referred directly to the palliative team. Second, the fragility test that we had decided to use to select the patients who would undergo a CGA is characterized by good sensitivity (83\%) at the cost of low specificity (50\%). That is, $50 \%$ of patients referred for a CGA because of a positive fragility test were not truly fragile according to the CGA. The establishment of an optimal fragility test for selecting patients for CGA has been an uphill battle in recent decades. ${ }^{15,16}$ There are various valid questionnaires, but to date there has been no consensus on which is the most useful in clinical practice. Some of the most widely used are the G8, ${ }^{28}$ the Triage Risk Screening Tool (TRST), ${ }^{29}$ and the Vulnerable Elders-13 Survey (VES-13). ${ }^{30}$ In our case, we deemed it important to use a test that had been validated in our own population and that had good sensitivity, accepting an excess of false positives, but our results question the suitability of such a tool as a method for optimizing healthcare resources. We do not believe that our results support the ineffectiveness of fragility tests in the elderly. In contrast, we think that they can be very useful in other healthcare areas where access to a CGA is more difficult and where it is not possible to include geriatricians on tumor committees. The choice of one fragility test over another should depend on each center's own experience.

Third, we found that the MCM modified the initial therapeutic plan in only 2 patients aged $70-79$ years $(6.6 \%)$. These results may be due mainly to the close collaboration between oncologists, geriatricians, and the palliative care team at our center, so that the initial assessment and treatment plan incorporated aspects that are only assessed by CGA in other environments. Thus, the frailty test and the CGA may have added little information to that taken into consideration when making an initial treatment plan. However, in octogenarian patients, MCM modified the treatment plan in $13.8 \%$ of the cases, that is, in one of every 7 patients. We think this makes a meaningful clinical difference compared with our younger sample. The prevalence of geriatric syndromes not detected without undergoing a CGA is probably higher in the octogenarian population. ${ }^{31,32}$ This fact would account for the impact of the model on the therapeutic decision in these patients. In our opinion, this influence is clinically relevant because it helps save resources and avoids iatrogenic risks and discomfort to our patients and their caregivers. We did not include quality-of-life questionnaires in the follow-up of our patients, though their interpretation would have been questionable in the absence of a control group.

Based on the results of this prospective observational study, the MCM for geriatric oncology patients was modified at the Consorci Sanitari de Terrassa, in the following ways: a geriatrician joined each of the main tumor committees (lung cancer, colorectal and gastric cancer, breast cancer, and urological cancer), and participates in the therapeutic approach for all patients older than 70 years; frailty screening tests are no longer performed; and only octogenarian patients who are initially candidates for active oncological treatment undergo CGA. In conclusion, an MCM in elderly oncologic patients, adapted to the circumstances of each center, is desirable and feasible in a general hospital, although various reasons often hinder patient recruitment for this kind of model. CGA can substantially modify the therapeutic plan for some of these patients, especially in the octogenarian population.

\section{Acknowledgments}

The authors thank Sylva Astrik Torossian (Corporació Sanitària Parc Tauli, Sabadell, Spain) and David Ambler for their linguistic support; Teresa Jimenez (Palliative Care Department, Consorci Sanitari de Terrassa, Terrassa, Spain) for the collection of data; and Daniel Fernández (Data Management Department, Consorci Sanitari de Terrassa) for data analysis and interpretation.

\section{References}

1. Abellán A, Pujol R. El estado de la población mayor en España, 2015. Blog Envejec [En-Red]. https://envejecimientoenred.wordpress.com/2015/01/26/el-estado-de-la-poblacion-mayor-enespana-2015/. Published January 2015. Accessed June 10th, 2016.

2. Ferlay J, E. Steliarova-Foucher E, Lortet-Tieulent J, et al. Cancer incidence and mortality patterns in Europe: Estimates for 40 countries in 2012. Eur J Cancer. 2013;49:1374-1403.

3. Quaglia A, Tavilla A, Shack L et al. The cancer survival gap between elderly and middle aged patients in Europe is widening. Eur J Cancer. 2009;45:1006-1016.

4. Petersen RC, Caracciolo B, Brayne C, et al. Mild cognitive impairment: a concept in evolution. J Intern Med. 2014;275(3):214-228.

5. Wildiers H, Highley MS, de Bruijn EA, van Oosterom AT. Pharmacology of anticancer drugs in the elderly population. Clin Pharmacokinet. 2003;42(14):1213-1242.

6. Klotz U. Pharmacokinetics and drug metabolism in the elderly. Drug Metab Rev. 2009;41(2):67-76.

7. Wedding U, Kődding D, Pientka L, Steinmetz HT, Schmitz S. Physicians' judgement and comprehensive geriatric assessment (CGA) select different patients as fit for chemotherapy. Crit Rev Oncol Hematol. 2007;64:1-9.

8. Hurria A, Togawa K, Mohile SG, et al. Predicting chemotherapy toxicity in older adults with cancer: a prospective multicenter study. J Clin Oncol. 2011;29:3457-3465.

9. Mislang A and Biganzoli L. Adjuvant systemic therapy in older breast cancer women: can we optimize the level of care? Cancers (Basel). 2015;7:1191-1214.

10. Repetto L, Fratino L, Audisio RA, et al. Comprehensive geriatric assessment adds information to Eastern Cooperative Oncology Group performance status in elderly cancer patients: an Italian 
Group for Geriatric Oncology study. J Clin Oncol. 2002;20:494-502.

11. Balducci L, Yates J. General guidelines for the management of older patients with cancer. Oncology. 2000;14:221-227.

12. Extermann M, Aapro M. Assessment of the older cancer patient. Hematol Oncol Clin North Am. 2000;14:63-77.

13. Sifer-Rivière L, Saint-Jean O, Gisselbrech M, et al. What the specific tools of geriatrics and oncology can tell us about the role and status of geriatricians in a pilot geriatric oncology program. Ann Oncol. 011;22:2325-2329.

14. Puts MT, Hardt J, Monette J, et al. Use of geriatric assessment for older adults in the oncology setting: a systematic review. J Natl Cancer Inst. 2012;104:1133-1163.

15. Decoster L, Van Puyvelde K, Mohile S et al. Screening tools for multidimensional health problems warranting a geriatric assessment in older cancer patients: an update on SIOG recommendations. Ann Oncol. 2015;26(2):288-300.

16. Hamaker ME, Jonker JM, de Rooij SE, Vos AG, Smorenburg CH, van Munster BC. Frailty screening methods for predicting outcome of a comprehensive geriatric assessment in elderly patients: a systematic review. Lancet Oncol. 2012;13:e437-e444.

17. Orfila F, Cegri F, Limón E. Fragilidad geriátrica en la consulta de atención primaria: validación predictiva de un instrumento. XXVII Congreso De Medicina De Familia Y Comunitaria. Aten Primaria. 2007;39(suppl 2):115-152.

18. Charlson ME, Pompei P, Ales KL, MacKenzie CR. A new method of classifying prognostic comorbidity in longitudinal studies: development and validation. J Chronic Dis. 1987;40(5):373-383

19. Gallagher P, Ryan C, Byrne S, Kennedy J, O’Mahony D. STOPP (Screening Tool of Older Person's Prescriptions) and START (Screening Tool to Alert doctors to Right Treatment). Consensus validation. Int J Clin Pharmacol Ther. 2008;46:72-83.

20. Lawton MP, Brody EM. Assessment of older people: Selfmaintaining and instrumental activities of daily living. Gerontologist. 1969; 9(3):179-186

21. Mahoney FI, Barthel DW. Functional evaluation: the Barthel Index. Md Med J. 1965;14:56-61.
22. Podsiadlo D; Richardson S. The timed 'Up \& Go': A test of basic functional mobility for frail elderly persons. J Am Geriatric Soc. 1991;39 (2):142-148.

23. Guralnik JM, Simonsick EM, Ferrucci L, et al. A short physical performance battery assessing lower extremity function: association with self-reported disability and prediction of mortality and nursing home admission. J Gerontol. 1994;49:M85-94.

24. Crum RM, Anthony JC, Bassett SS, Folstein MF. Population-based norms for the Mini-Mental State Examination by age and educational level. JAMA. 1993;269(18):2386-2391.

25. Kirby M, Denihan A, Bruce I, Coakley D, Lawlor BA. The clock drawing test in primary care: sensitivity in dementia detection and specificity against normal and depressed elderly. Int J Geriatr Psychiatry. 2001;16:935-940.

26. Greenberg SA. How to try this: the Geriatric Depression Scale: Short Form. Am J Nurs. 2007;107(10):60-69.

27. Rubenstein LZ, Harker JO, Salva A, Guigoz Y, Vellas B. Screening for undernutrition in geriatric practice: developing the ShortForm Mini Nutritional Assessment (MNA-SF). J Geront. 2001;56A:M366-377.

28. Bellera C, Rainfray M, Mathoulin-Pélissier S et al. Screening older cancer patients: first evaluation of the G-8 screening tool. Ann Oncol. 2012; 23:2166-2172.

29. Meldon SW, Mion LC, Palmer RM et al. A brief risk-stratification tool to predict repeat emergency department visits and hospitalizations in older patients discharged from the emergency department. Acad Emerg Med. 2003;10:224-232.

30. Saliba D, Elliot M, Rubenstein LZ et al. The Vulnerable Elders Survey: a tool for identifying vulnerable older people in community. J Am Geriatric Soc. 2001;49:1691-1699.

31. Davies K, Jagger C, Kingston A, et al. Health and disease in 85 year olds: baseline findings from the Newcastle $85+$ cohort study. BMJ. 2009;339:b4904.

32. Piccirillo JF, Vlahiotis A, Barrett LB, et al. The changing prevalence of comorbidity across the age spectrum. Crit Rev Oncol Hematol. 2008;67:124-132. 